\title{
Four pulse sequences necessary for liver MRI interpretation
}

Linda Pantongrag-Brown, M.D.

From Advanced Diagnostic Imaging Center, Ramathibodi Hospital, Bangkok, Thailand.

Address correspondence to L.P. (e-mail: 1brown800@gmail.com)

Keywords: Liver MRI, Pulse sequences, Liver mass.

MRI is the best imaging modality for detection and characterization of liver masses. There are multiple pulse sequences used in MRI and they can be confusing and difficult to understand. Therefore, four important pulse sequences are introduced in this article in order to simplify the seemingly complex pulse sequences, and allow general radiologists and clinicians of all specialties to approach MRI of liver masses with ease.

\section{Pulse sequence 1: T1 inphase/outphase (Figure 1)}

This sequence is used to determine fat content. If the image voxel contains both water and fat, the signal will enhance in the T1 inphase and decrease in the T1 outphase. Fatty liver, focal fatty infiltration, and fat-sparing lesions can be diagnosed with this pulse sequence.
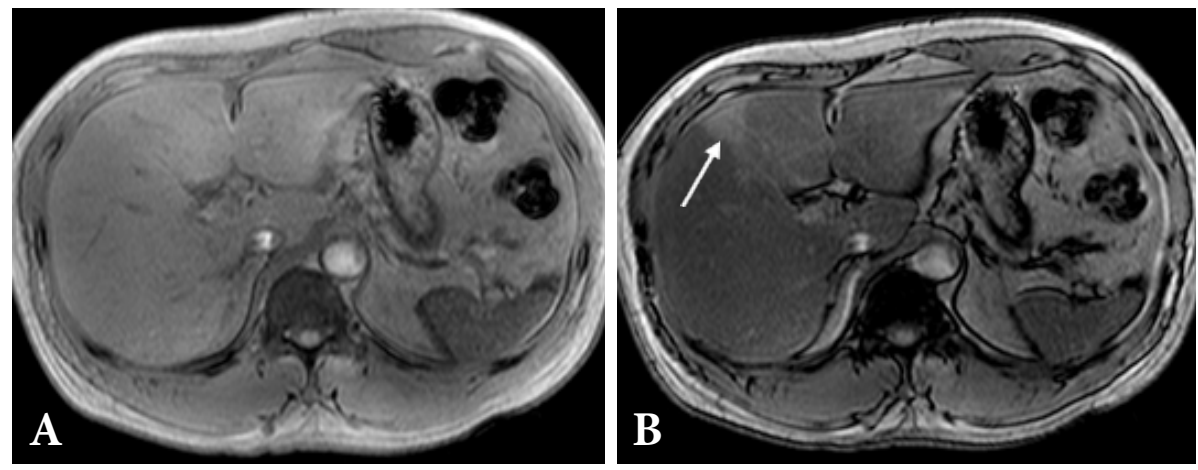

Figure 1: T1 inphase/outphase sequence

Fatty liver shows signal dropping at T1 outphase (B), compared to T1 inphase (A), except for an area of focal fatty sparing (arrow in B). 


\section{Pulse sequence 2: T2 and heavy T2 fat saturation (Figure 2)}

T2 fat saturation (T2 FS) is a pulse sequence to determine if the lesion is a true or pseudo lesion. Most true lesions will exhibit high signal intensity (SI); exceptions exist, such as early hepatocellular carcinoma and focal nodular hyperplasia, which may show similar intensity to the liver. Heavy T2 is used to distinguish solid from cystic lesions and hemangiomas. Cysts and hemangiomas will show very bright SI, similar to spinal fluid, whereas solid lesions will show not-so-bright SI but just a higher signal than that of liver parenchyma.

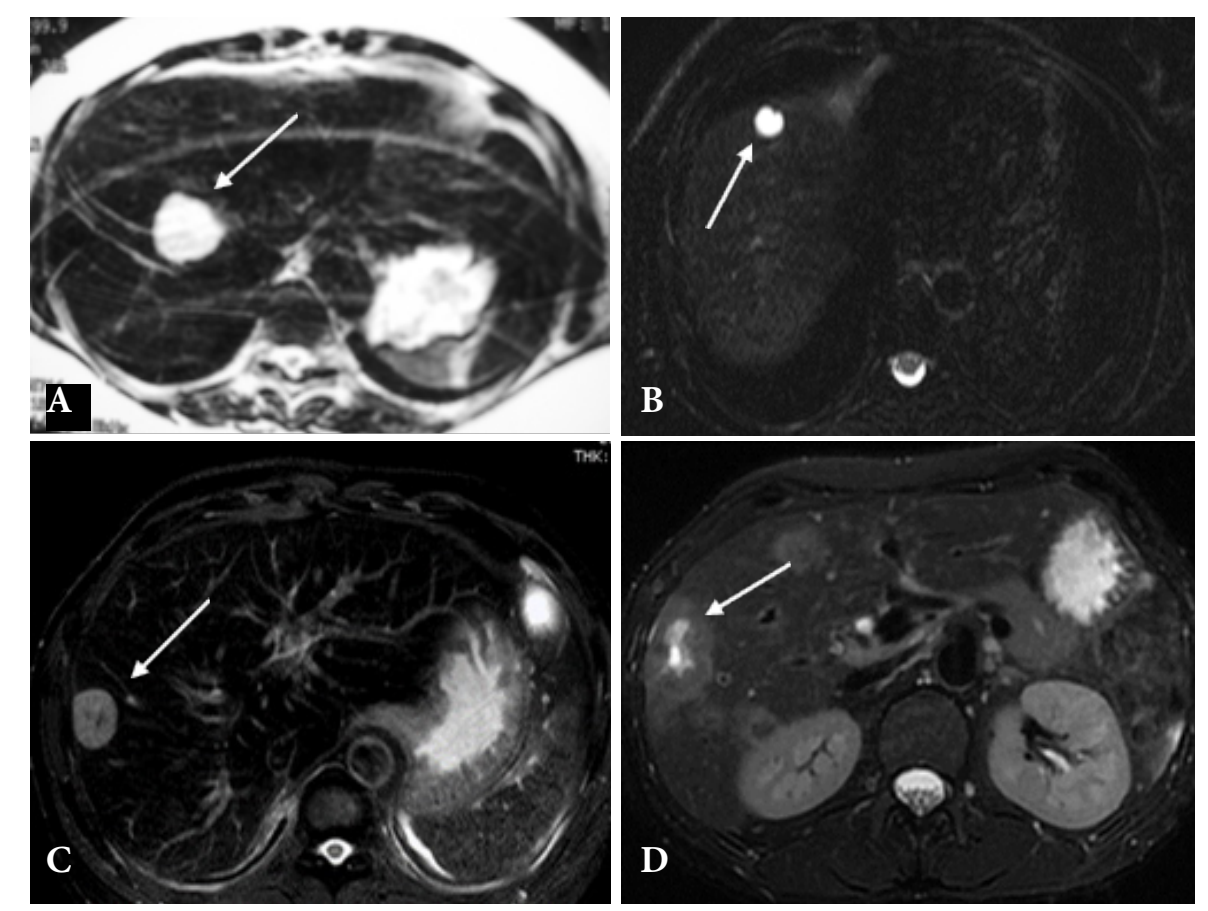

Figure 2: $\mathrm{T} 2$ and heavy $\mathrm{T} 2$ fat saturation sequence

Liver hemangioma (A), and liver cyst (B) show very bright SI at T2, whereas hepatoma (C) and metastasis $(D)$ show not-so-bright SI, but just a higher signal than the liver parenchyma.

\section{Pulse sequence 3: T1 dynamic gadolinium (Figure 3)}

This is the most important pulse sequence for liver mass analysis. Vascular enhancement pattern is the key to characterizing each mass. Dynamic contrast study, with arterial, venous and delayed phases, is mandatory for the evaluation. Each tumor has its own characteristic pattern of vascular enhancement, although overlapping may occur. 
If gadoxetic acid (Primovist), the hepatobiliary-specific contrast agent, is used, a 20 min delayed phase has to be evaluated after the dynamic phases. The liver parenchyma needs about 10-20 min to uptake contrast media and exhibit high SI. Most tumors show no uptake of gadoxetic acid. Generally, uptake of gadoxetic acid by a liver mass indicates benign nature. For example, uptake is seen with focal nodular hyperplasia (FNH), nodular regenerative hyperplasia ( $\mathrm{NRH})$, benign regenerating and low-grade dysplastic nodules.

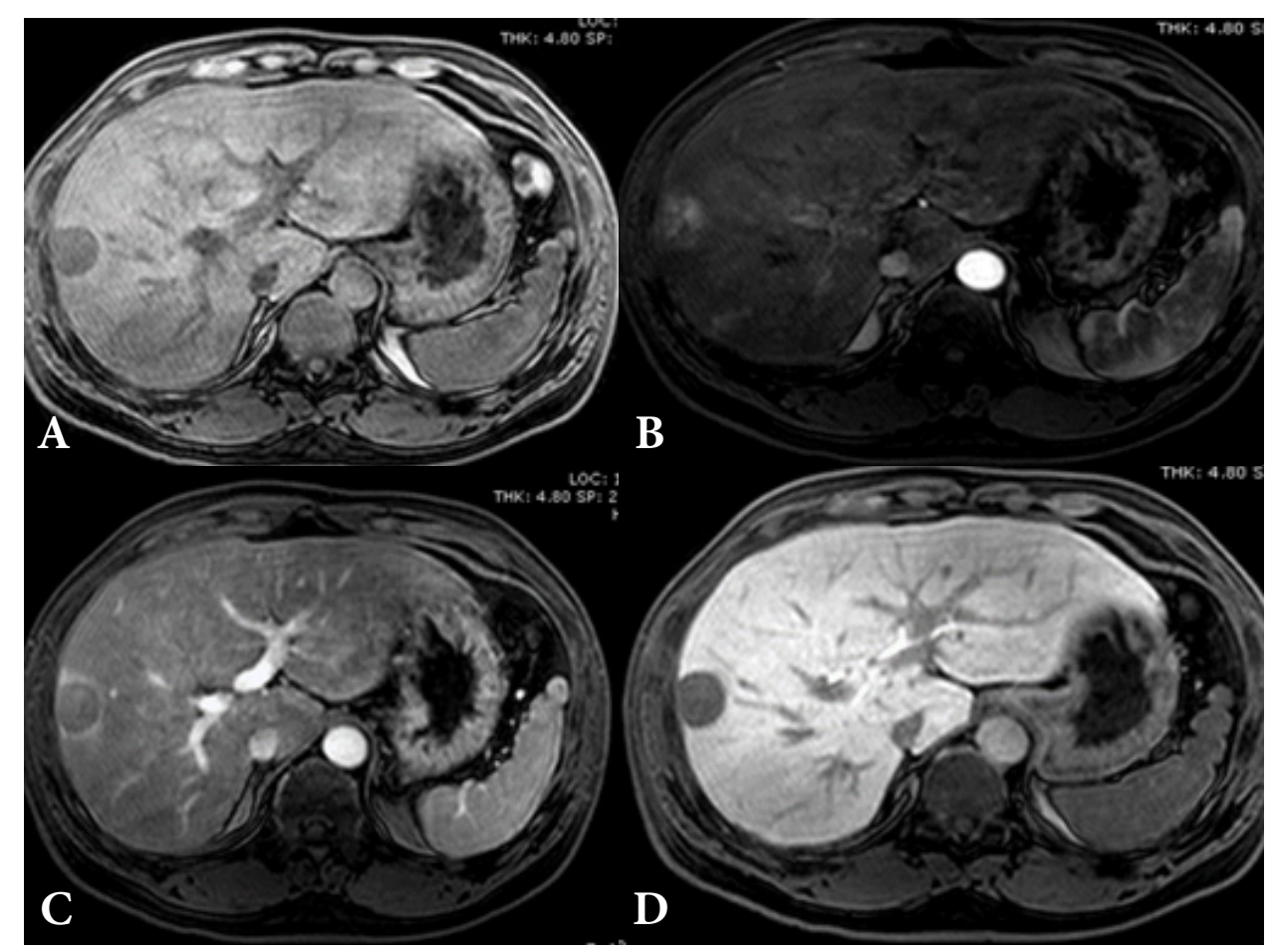

Figure 3: T1 dynamic gadoxetic acid contrast with 20 min delayed hepatobiliary (HB) phase of a small hepatocellular carcinoma

A: Pre-contrast shows a low SI nodule.

B: Arterial phase shows rapid enhancement of the nodule.

$C$ : Venous phase shows rapid washout of the nodule.

D: 20 min HB phase shows no uptake of gadoxetic acid by the nodule. Note that the liver parenchyma shows high SI which is secondary to uptake of gadoxetic acid by normal hepatocytes. 


\section{Pulse sequence 4: Diffusion weighted image (Figures 4-5)}

Diffusion weighted image (DWI) is a complimentary pulse sequence and not necessary for analyzing liver masses. It is based upon measuring the random Brownian motion of water molecules within a voxel of tissue. The relationship between histology and diffusion is complex. In general, highly cellular tissues or those with cellular swelling exhibit lower diffusion coefficients, and thus show abnormal fluid restriction. A low B value is sensitive but not specific, whereas a high $B$ value is specific but not sensitive. Therefore, low and high $B$ values are used in abdominal MRIs (B 0-50 for low, and B 500-1000 for high). DWI is a T2-based image, therefore T2 shine-through effect may mimic abnormal fluid restriction. When in doubt, combining with an ADC map is recommended. T2 shine-through effect will produce high $\mathrm{ADC}$ value, whereas true restriction will produce low $\mathrm{ADC}$ value.

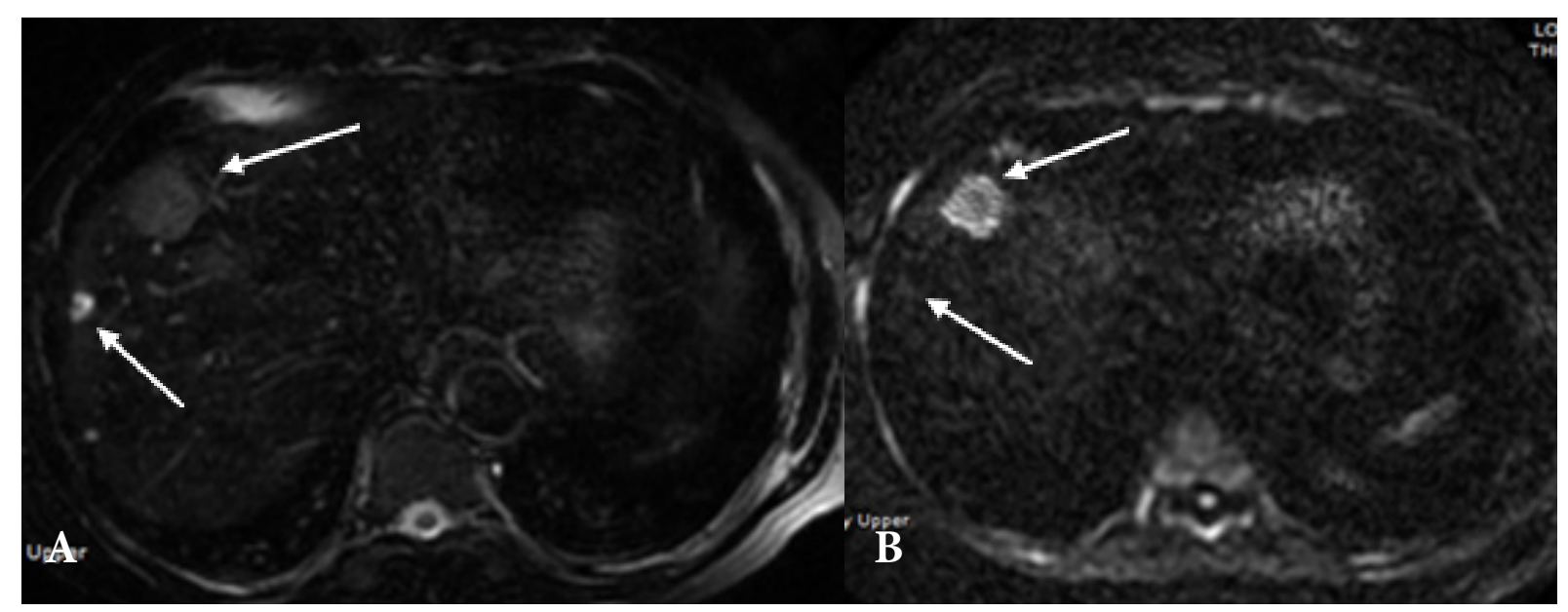

Figure 4: Diffuse weighted image (DWI), B 1000

Two lesions in the liver are demonstrated. The large nodule is a metastatic nodule, and the small nodule is a simple cyst. The large, metastatic nodule shows slightly high SI at T2 (A) with fluid restriction at DWI (B: seen as high SI). The small, simple cyst shows very high SI at T2 without fluid restriction at DWI (B: seen as isointense to the liver). 


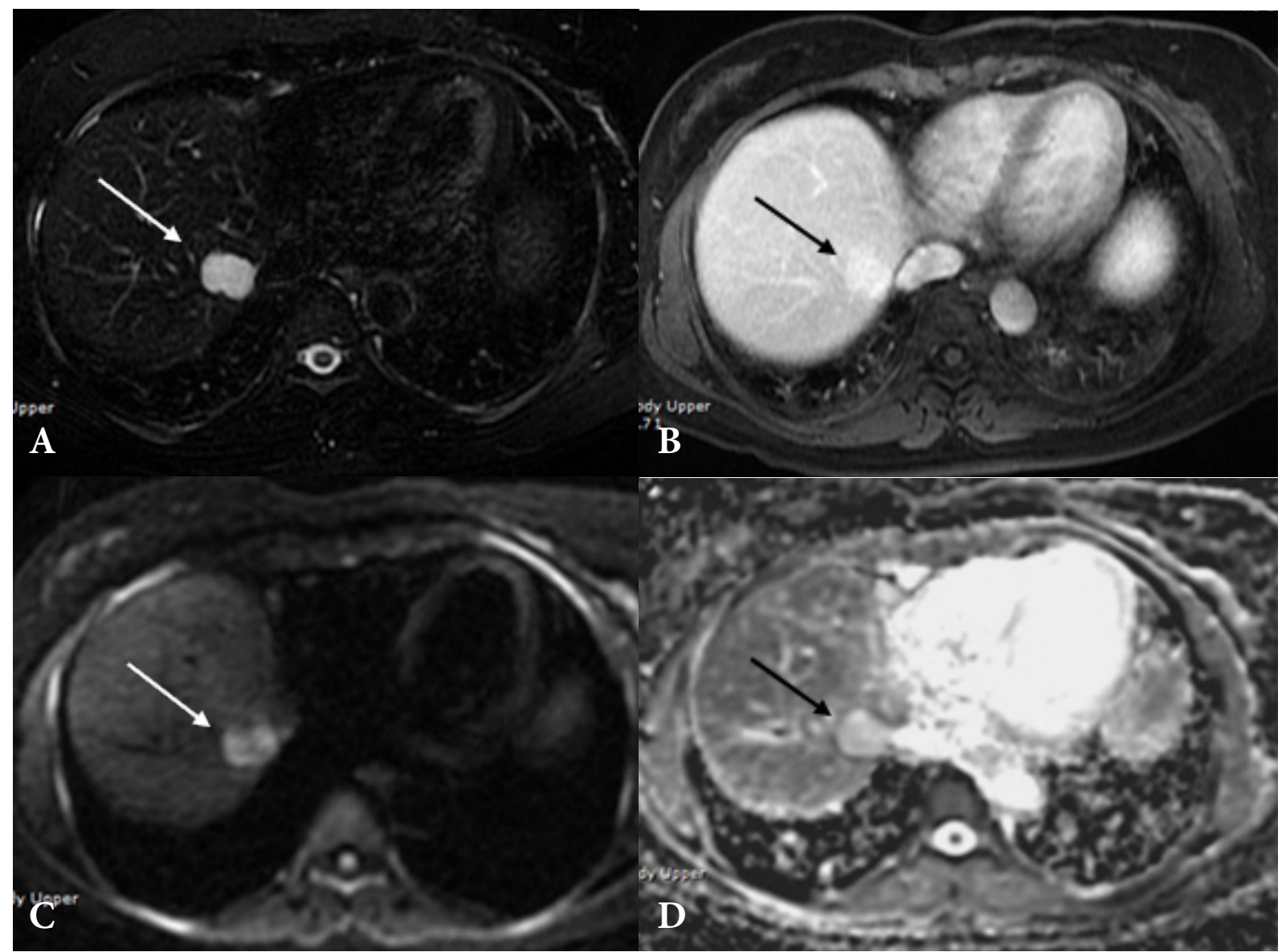

Figure 5: DWI and T2 shine-through effect

A hemangioma shows very high SI at T2 FS (A), and persistent enhancement in the delayed phase of contrast enhancement (B). At DWI (B 1000), the nodule shows high SI because of T2 shinethrough effect $(C)$, which is confirmed by high SI in the ADC map (D).

\section{Case Examples}

Following are examples of common liver lesions and the application of the above-mentioned pulse sequences for analysis. Each liver nodule, mass, and pseudo lesion exhibit a different MRI pattern [1-7]. Images of the lesions are shown and MRI patterns are interpreted using the summarized guidelines below. 


\section{Focal fatty infiltration (Figure 6)}

\begin{tabular}{ll} 
MRI signal & Interpretation \\
\hline T1 inphase/outphase & Signal loss = fat \\
T2 FS & Low SI = pseudo lesion \\
T1 dynamic gadolinium & No enhancement, normal vessels coursing through
\end{tabular}

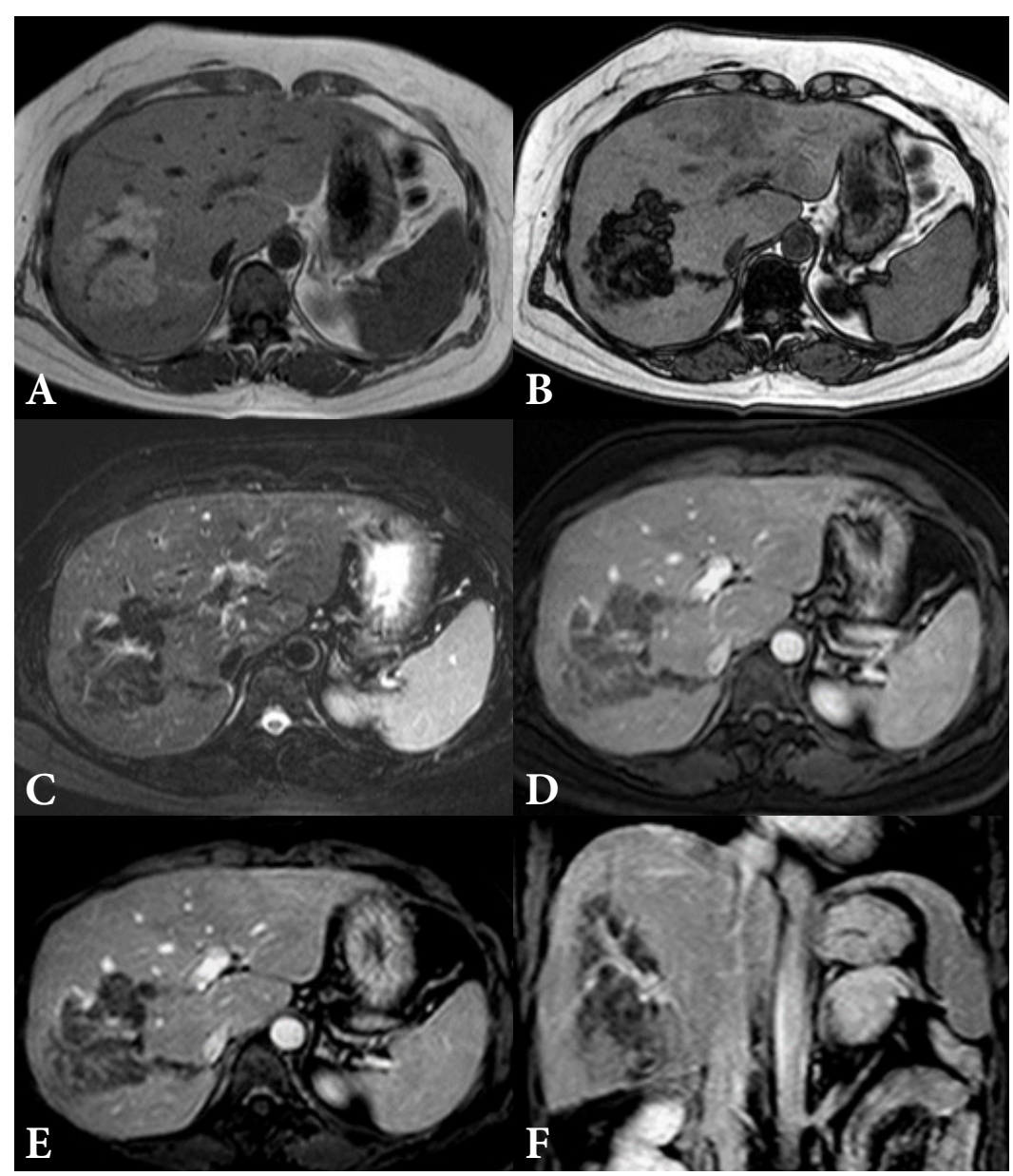

Figure 6: Focal fatty infiltration in an asymptomatic female

A, B: T1 inphase (A), and outphase (B) show a lobulated mass at right hepatic lobe with signal loss, indicative of a fat containing mass.

C: T2 FS shows the lesion to be low SI, suggestive of a pseudo lesion.

$D$-F: Arterial (D), venous (E), and delayed (F) phases show no enhancement within the lesion. Note the vessel coursing through this pseudo lesion, which is diagnostic for focal fatty infiltration. 


\section{Hemangioma (Figure 7)}

\begin{tabular}{ll} 
MRI signal & Interpretation \\
\hline T1 inphase/outphase & $\begin{array}{l}\text { No signal loss = no fat } \\
\text { High SI = true lesion, very bright signal similar to } \\
\text { spinal fluid }\end{array}$ \\
$\begin{array}{l}\text { T1 dynamic gadolinium } \\
\text { and } 3 \text { min delay }\end{array}$ & $\begin{array}{l}\text { Peripheral nodular enhancement, central filling-in, } \\
\text { and persistent enhancement throughout delayed } \\
\text { phase }\end{array}$
\end{tabular}

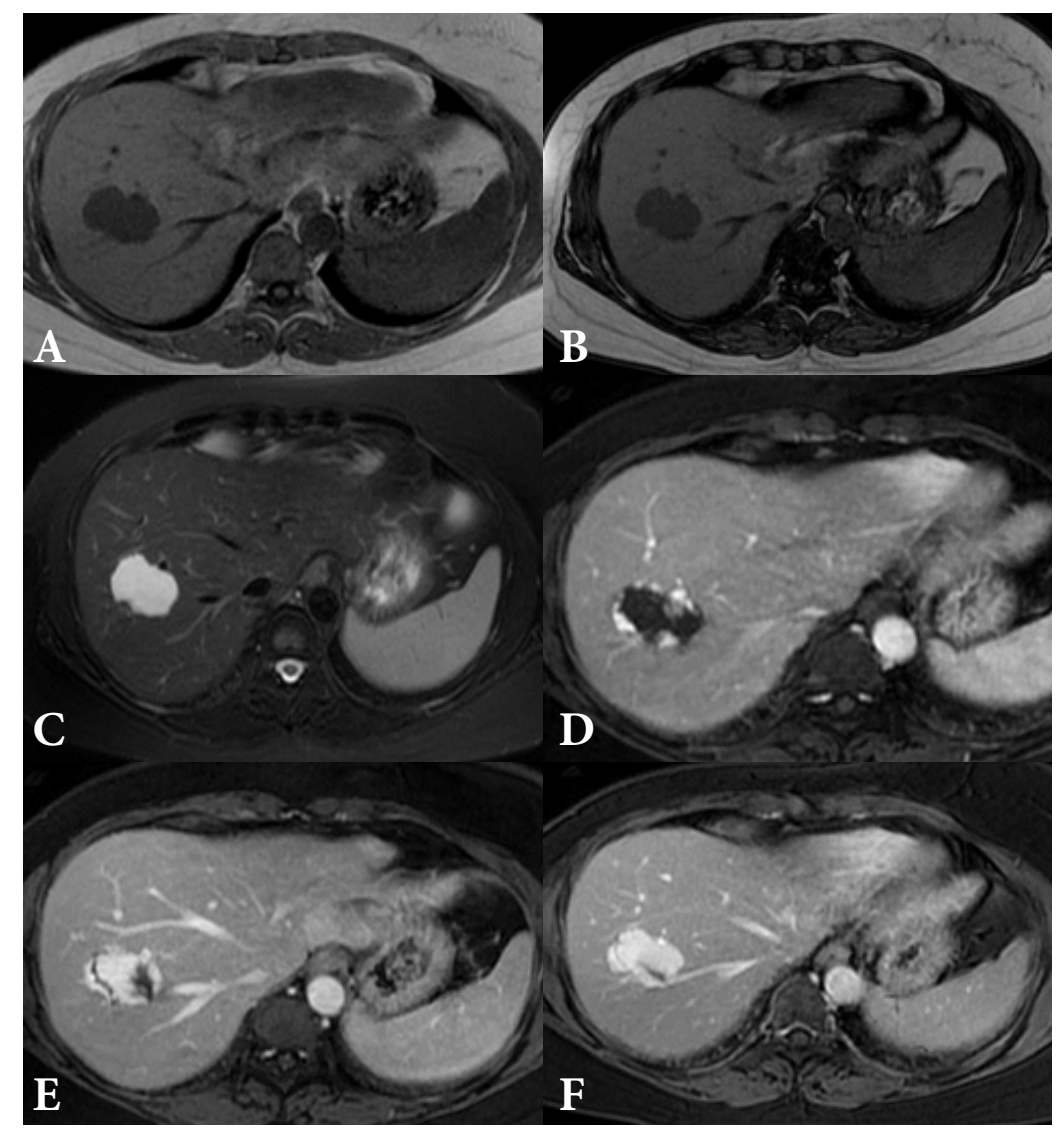

Figure 7: Hemangioma in a woman with breast cancer

A, B: T1 inphase/outphase shows a low SI nodule in the right hepatic lobe.

C: T2 FS shows the nodule to be high SI, similar to spinal fluid.

$D$-F: The nodule shows peripheral nodular enhancement at arterial phase (D), central filling-in at venous phase (E), and persistent enhancement throughout delayed phase (F). MRI pattern is characteristic of a benign hemangioma. 


\section{Focal nodular hyperplasia (Figure 8)}

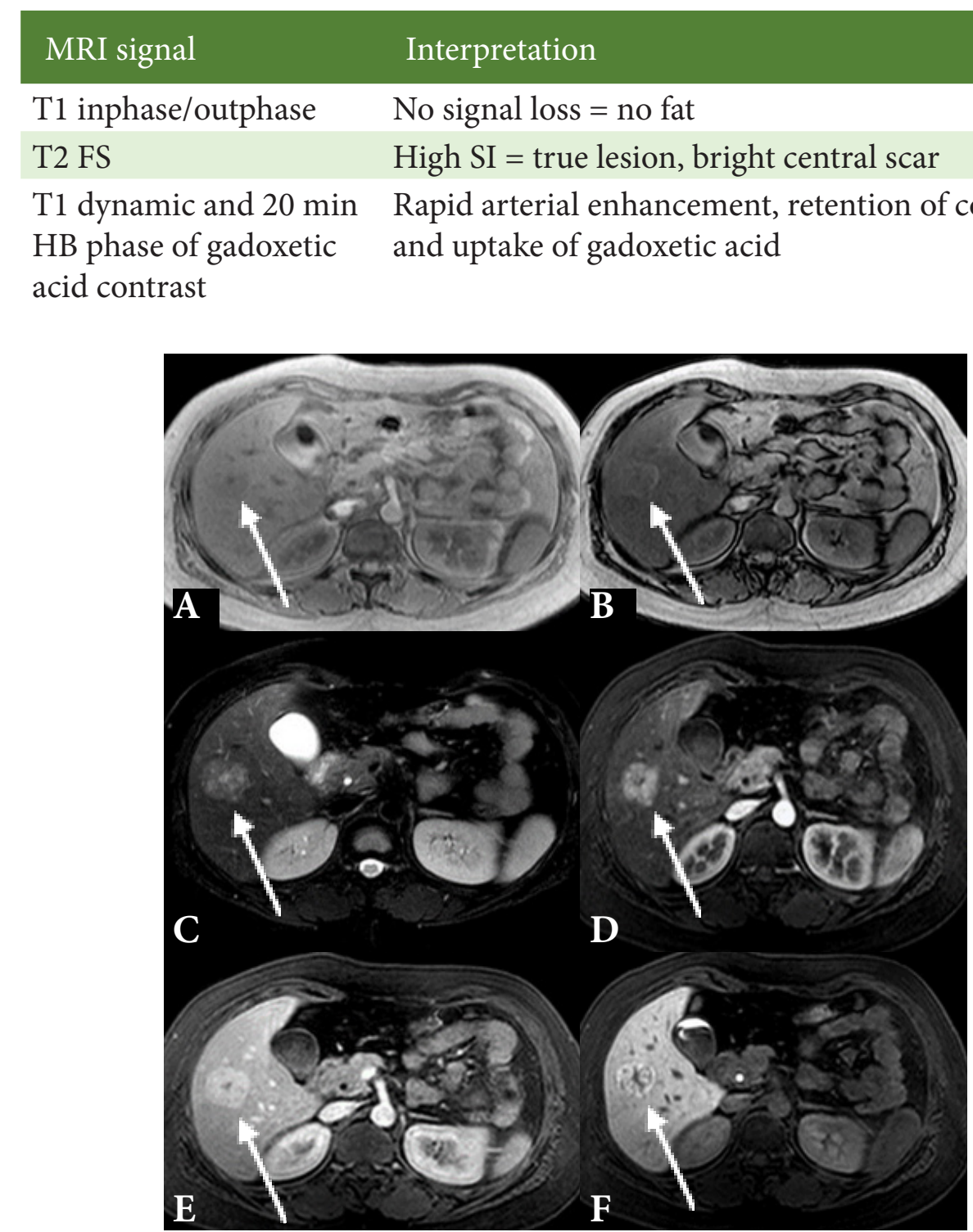

Figure 8: Focal nodular hyperplasia (FNH) in an asymptomatic woman

$A, B:$ T1 inphase/outphase show fatty liver, as demonstrated by signal loss at outphase (B) compared to inphase (A). A lobulated mass at right hepatic lobe is noted.

C: T2 FS shows the nodule to be high SI with a very bright small central scar.

$D$-F: The nodule shows rapid arterial enhancement (D), retention of contrast at venous phase (E), and uptake of gadoxetic acid at HB phase [except for a central scar (F)]. This MRI pattern is characteristic of a benign FNH. 


\section{Hepatocellular carcinoma (Figure 9)}

$\begin{array}{ll}\text { MRI signal } & \text { Interpretation } \\ \text { T1 inphase/outphase } & \text { No signal loss = no fat } \\ \text { T2 FS } & \text { High SI = true lesion } \\ \text { T1 dynamic and 20 min } & \begin{array}{l}\text { Rapid arterial enhancement, rapid venous washout, } \\ \text { and no uptake of gadoxetic acid } \\ \text { HB phase of gadoxetic } \\ \text { acid contrast }\end{array} \\ \text { DWI } & \text { High SI = fluid restriction } \\ \text { ADC map } & \text { Low SI = confirms fluid restriction }\end{array}$

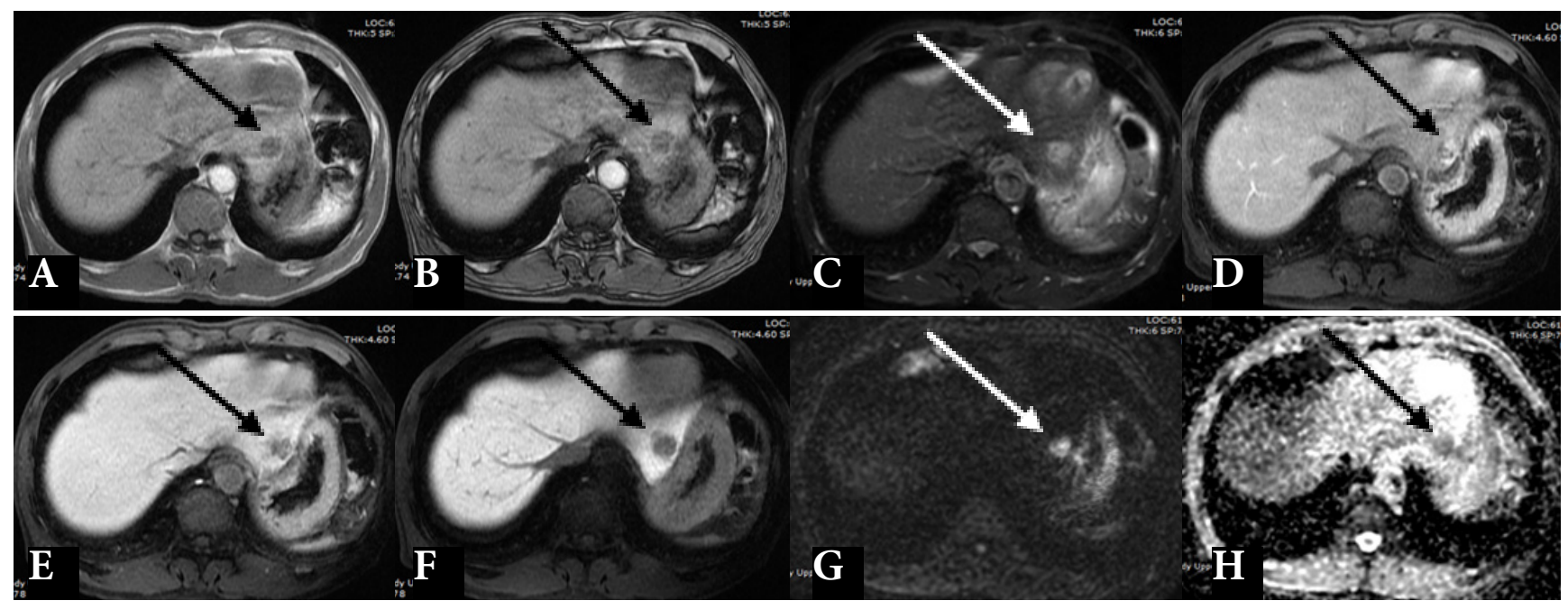

Figure 9: HCC in a man with chronic hepatitis B

A, B: T1 inphase/outphase show a $1.6 \mathrm{~cm}$ nodule at segment 2 of the left hepatic lobe.

C: T2 FS shows the nodule to be high SI, but not as bright as fluid.

$D-F$ : The mass shows rapid arterial enhancement $(D)$, rapid venous washout $(E)$, and no uptake of gadoxetic acid contrast (F). This MRI pattern is characteristic of HCC.

G-H: The mass shows high SI at DWI, B 1000 (G) and low SI at ADC map (H), confirming the diagnosis of HCC. 


\section{Conclusions}

1. MRI of the liver can be simplified in 4 key pulse sequences. Four key MRI pulse sequences are T1 inphase/outphase, T2 and heavy T2 FS, T1 dynamic gadolinium with $20 \mathrm{~min} \mathrm{HB}$ phase (if gadoxetic acid contrast is used), and DWI.

2. T1 inphase/outphase is to determine fat content.

3. T2 is to differentiate true lesion from pseudo lesion.

4. T1 dynamic gadolinium is to determine vascular enhancement patterns. This is the most important pulse sequence. $20 \mathrm{~min} \mathrm{HB}$ phase of gadoxetic acid is used to confirm a diagnosis of $\mathrm{FNH}$, and to distinguish a dysplastic nodule from HCC.

5. DWI is a complimentary pulse sequence. A nodule with a highly cellular component usually shows fluid restriction at DWI. 


\section{References}

1. Buetow PC, Pantongrag-Brown L, Buck JL, Ros PR, Goodman ZD. Focal nodular hyperplasia of the liver: radiologic-pathologic correlation. Radiographics 1996;16:369-88.

2. Hussain SM, Terkivatan T, Zondervan PE, Lanjouw E, de Rave S, Ijzermans JN, et al. Focal nodular hyperplasia: findings at state-of-the-art MR imaging, US,CT, and pathologic analysis. Radiographics 2004;24:3-9.

3. Pantongrag-Brown L. Imaging of focal liver masses. Thai J Gastroenterol 2004;5:130-6.

4. Pantongrag-Brown L. Multiple faces of focal nodular hyperplasia. Thai J Gastroenterol 2007;8:139-43

5. Pantongrag-Brown L. Imaging approach to liver mass. Part 1: Incidental finding without underlying liver disease. Thai J Gastroenterol 2008;9:43-7.

6. Pantongrag-Brown L. Imaging approach to liver mass. Part 2: Liver mass with underlying chronic liver disease. Thai J Gastroenterol 2008;9:113-6.

7. Anderson SW, Kruskal JB, Kane RA. Benign hepatic tumors and iatrogenic pseudotumors. Radiographics 2009;29:211-29. doi:10.1148/rg.291085099. 